\title{
Multiply-charged cations: remarkable structures and stabilities
}

\author{
Leo Radom, Peter M.W. Gill, Ming Wah Wong and Ross H. Nobes \\ Research School of Chemistry, Australian National University, Canberra, A.C.T. 2601, \\ Australia
}

\begin{abstract}
The use of ab initio molecular orbital theory with moderately large basis sets and incorporation of electron correlation to study multiply-charged ions is discussed. Despite extreme coulomb repulsion, several of the ions have very short bonds. The $\mathrm{C}-\mathrm{F}$ lengths in $\mathrm{HCF}^{2+}(1.111 \dot{\mathrm{A}})$ and $\mathrm{HeCF}^{3+}(1.102 \dot{\mathrm{A}})$ and the $\mathrm{C}-\mathrm{Cl}$ length in $\mathrm{HCCl}^{2+}(1.466 \dot{\mathrm{A}})$ are the shortest known for these types of bonds. Some of the more highly charged ions which have been examined, including the $\mathrm{HeCF}^{3+}$ and $\mathrm{CHe}_{3}{ }^{3+}$ trications and the $\mathrm{CHe}_{4}{ }^{4+}$ tetracation, are predicted to be observable species, despite the possibility of highly exothermic (by $\sim 1000 \mathrm{~kJ} \mathrm{~mol}^{-1}$ and more) fragmentations. It is found that unrestricted Moller-Plesset perturbation theory is not a suitable procedure for examining the fragmentation of multiply-charged ions in cases where spin contamination is significant in the underlying unrestricted Hartree-Fock wavefunction. Transition structures for asymmetric fragmentations of multiply-charged ions often occur late on the reaction pathway, with the length of the breaking bond two or more times greater than its value in the corresponding equilibrium structures.
\end{abstract}

\section{INTRODUCTION}

There has been considerable recent interest in the gas-phase chemistry of multiply-charged cations. Part of the impetus for studying multiply-charged ions has come from experiment, where recent advances have permitted increasingly detailed characterization of such species. Methods which have been applied include charge-stripping, double-charge-transfer, photon double ionization and Auger spectroscopy (refs 1-4). The impetus has also come from theory (for recent reviews, see refs 5-7). Theoretical calculations can be used to advantage to study multiply-charged ions, since they can be applied as readily to short-lived species as to normal stable molecules.

This presentation is directed partly towards general problems which may be encountered in calculations on multiply-charged ions and partly towards specific multiply-charged systems which we have found to be of interest. Most of the previous theoretical work on multiply-charged ions has been restricted to dications (refs 5-7). Much of what we have done in this area has also been directed at dications (see, for example, refs 815) but we have also been especially interested in more highly charged ions - trications and tetracations (see. for example, refs 16-18) - and some of our predictions for these species are also discussed below.

\section{METHOD}

Full details of the methods used for the various studies described herein are given elsewhere (refs 13-18). Briefly, ab initio molecular orbital calculations (ref. 19) have been carried out with a variety of basis sets (typically $6-31 \mathrm{G}(\mathrm{d}), 6-31 \mathrm{G}(\mathrm{d}, \mathrm{p})$ or $6-311 \mathrm{G}(\mathrm{d}, \mathrm{p})$ ) and with electron correlation normally incorporated using Møller-Plesset perturbation theory. The Møller-Plesset calculations have been carried out both with a restricted Hartree-Fock (RHF) starting point (leading to restricted Møller-Plesset or RMP results) and with an unrestricted Hartree-Fock (UHF) starting point (leading to UMP results). Vibrational frequencies have been routinely calculated in order to characterize stationary points as minima (representing equilibrium structures) or saddle points (representing transition structures). Throughout this paper, bond lengths are in angströms and bond angles in degrees. 


\section{FRAGMENTATION OF DICATIONS: DECEPTIVE CONVERGENCE IN MøLLER-PLESSET PERTURBATION ENERGIES}

Dications can typically fragment to two monocations in a highly exothermic manner. Whether or not a particular dication is potentially observable depends on the magnitude of the barrier to fragmentation. It is important, therefore, to be able to calculate such barriers reliably but with standard procedures this may present difficulties.

In order to examine this problem in detail, we have carried out calculations on one of the simplest dications, $\mathrm{He}_{2}{ }^{2+}$ (ref. 13). There have been numerous previous theoretical studies on this species, which is isoelectronic with $\mathrm{H}_{2}$ (see, for example, ref. 20). It was observed experimentally for the first time in 1984 (ref. 21).

The $\mathrm{He}_{2}{ }^{2+}$ dication has the shortest known bond length $(0.703 \AA$ ) despite having an exothermicity for fragmentation to two $\mathrm{He}^{+\cdot}$ monocations of $836 \mathrm{~kJ} \mathrm{~mol}^{-1}$ (ref. 20). The fact that $\mathrm{He}_{2}{ }^{2+}$ is observable is consistent with a significant barrier to this fragmentation.

Fragmentation barriers for $\mathrm{He}_{2}{ }^{2+}$ calculated with a large basis set at the restricted Hartree-Fock and Moller-Plesset levels (Table 1) appear to show poor convergence. There is a continuing large variation in going from RHF (805) to RMP2 (345) to RMP3 (210) to RMP4 $\left(157 \mathrm{~kJ} \mathrm{~mol}^{-1}\right)$. This is not particularly surprising: RHF theory is well known to dissociate incorrectly in the case of $\mathrm{H}_{2}$ so it may already be in difficulty at the transition structure for dissociation in $\mathrm{He}_{2}{ }^{2+}$.

TABLE 1. Calculated barrier heights ${ }^{\mathrm{a}}$ for homolytic fragmentation of $\mathrm{He}_{2}{ }^{2+}$

\begin{tabular}{llllc}
\hline RHF & RMP2 & RMP3 & RMP4 & Full CI \\
805 & 345 & 210 & 157 & 139 \\
\hline & & & & \\
UHF & UMP2 & UMP3 & UMP4 & Full CI \\
166 & 251 & 266 & 266 & 139 \\
\hline
\end{tabular}

a Calculated with a [3s3p1d] basis set, from ref. 13.

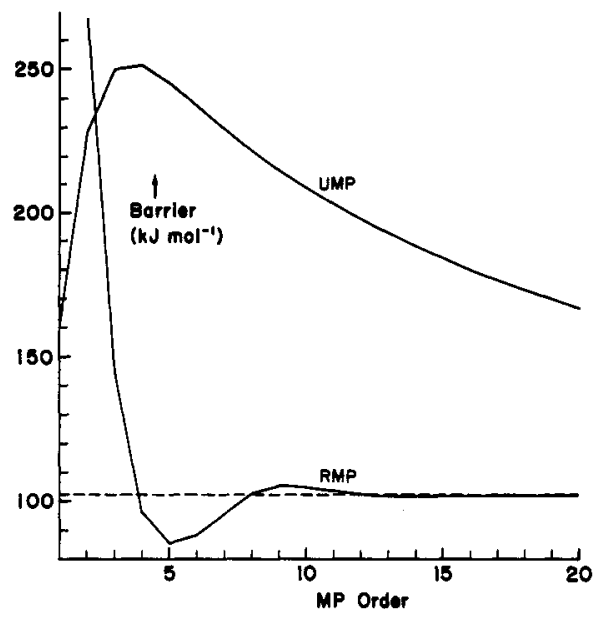

Fig. 1 Barriers to homolytic fission of $\mathrm{He}_{2}{ }^{2+}$ as calculated using MPn theory $(n=1-20)$ and a minimal basis set (from ref. 13).

On the other hand, unrestricted Hartree-Fock theory is well known to yield the correct dissociation energy in the case of $\mathrm{H}_{2}$. Accordingly, the barrier values that are obtained at the UMP levels (also included in Table 1) appear to have converged much better than the RMP results: 166 at UHF, 251 at UMP2, 266 at UMP3 and $266 \mathrm{~kJ} \mathrm{~mol}^{-1}$ at UMP4.

Unfortunately, this apparent convergence is deceptive. When we calculate the exact barrier height for this basis set, obtained through full configuration interaction $(\mathrm{CI})$ calculations, we find that it is $139 \mathrm{~kJ} \mathrm{~mol}^{-1}$, a long way from $266 \mathrm{~kJ} \mathrm{~mol}^{-1}$. This deceptive convergence is rather worrying because our normal criterion for knowing to what extent to believe an MP4 result is based on the variation in going from MP2 to MP3 to MP4. We would have reached the wrong conclusion in this case.

We extended the Møller-Plesset treatment to higher orders of perturbation theory (e.g. MP5, MP10, etc) through calculations with a minimal basis set (ref. 13). Fortunately, the minimal basis set mimics quite well the behavior of MP theory with large basis sets. We can see (Fig. 1) that convergence with RMP theory although initially erratic, is rapid, so-called chemical accuracy being achieved above RMP7. Convergence of the UMP series, on the other hand, is extremely slow, and even at UMP20 there is an error of more than 60 $\mathrm{kJ} \mathrm{mol}^{-1}$ in the estimated barrier height. 
The problems with the UMP2 treatment appear to be associated with spin contamination in the UHF wavefunction for stretched bonds. It is important to realize that, while the UHF wavefunction dissociates correctly in an energetic sense, the wavefunction itself becomes a progressively poorer and poorer approximation as the bond length increases and therefore provides an increasingly poor starting point for a Meller-Plesset perturbation treatment. Related observations have been made by Handy and by Schlegel and their co-workers (refs 22,23 ). The results that we have obtained are indicative of dangers inherent generally in using the UMP approach with significantly spin-contaminated wavefunctions (see, for example, ref. 24).

\section{FRAGMENTATION OF DICATIONS: UNUSUALLY LONG-BONDED TRANSITION STRUCTURES}

Dications such as $\mathrm{He}_{2}{ }^{2+}$ are stable because, as the $\mathrm{He}---\mathrm{He}$ internuclear separation is stretched from its equilibrium value, the initial loss in covalent binding energy (an exponential decay) is greater than the decrease in energy due to decreased coulomb repulsion (a $1 / \mathrm{r}$ effect). The transition structure for fragmentation in fact corresponds to the point at which the rates of change of covalent binding and coulomb repulsion balance.

The internuclear separation in the transition structure for a symmetric fragmentation is typically $\sim \mathbf{5 0 \%}$ greater than in the equilibrium structure. For example, for the fragmentation of $\mathrm{He}_{2}{ }^{2+}$, the bond length in the transition structure is $1.15 \AA$ compared with $0.70 \AA$ in the equilibrium structure. In asymmetric fragmentations. on the other hand, the bond length in the transition structure is often two or more times greater than that in the equilibrium structure. For example, for the deprotonation of $\mathrm{AlH}^{2+}$ the relevant lengths are 3.59 and $1.65 \AA$. respectively $(\mathrm{UHF} / 6-31 \mathrm{G}(\mathrm{d}))$. An even longer bond is found in the transition structure for fragmentation of $\mathrm{MgH}^{2+}(12.45 \AA)$.

Such results are surprising because they imply that the balance between the rates of change of covalent binding and coulomb repulsion is not achieved until quite large internuclear separations in these cases, at which stage the covalent binding must surely be very small. What is the explanation for this puzzling state of affairs?

An electrostatics-based explanation (ref. 14) begins by recognizing that the coulomb repulsion energy in an asymmetric dication $A B^{2+}$ is not $1 / r$ but is $q_{A} q_{B} / r$. For example, in the case of $A^{2} H^{2+}$, at the equilibrium structure the calculated charges are $\mathrm{q}_{A} \sim 1.7$ and $\mathrm{q}_{B} \sim 0.3$ so that $\mathrm{q}_{A} \mathrm{q}_{B} / \mathrm{r} \sim 0.5 / \mathrm{r}$. As $\mathrm{r}$ increases, $\mathrm{q}_{A}$ and $\mathrm{q}_{B}$ both approach unity so that both the numerator and denominator in $q_{A} q_{B} / r$ increase. The coulomb repulsion therefore does not decrease as $1 / \mathrm{r}$ initially - it might even increase (Fig. 2). The consequence is that the point at which the rate of decay of coulomb repulsion matches the rate of decay of covalent binding (the transition structure) may occur much later than might initially have been anticipated.

We find that the electrostatics argument has only limited applicability. For many systems, the charges $q_{A}$ and $q_{B}$ tend to 2.0 and 0.0 , respectively, as the $A-B$ bond is stretched initially from its equilibrium length. This is the situation, for example, for $\mathrm{MgH}^{2+}$, and suggests that at the shorter internuclear separations the $\mathrm{MgH}^{2+} \cdot$ potential function resembles that of $\mathrm{Mg}^{2+}+\mathrm{H}^{\cdot}$ and there is little coupling with the $\mathrm{Mg}^{+}+\mathrm{H}^{+}$ diabatic curve (see below). The charges reach unity only in the vicinity of the transition structure $(12.45 \AA)$. In cases like these, a prediction of the transition-structure bond length cannot be made on the basis of electrostatic arguments alone and an alternative approach is necessary.

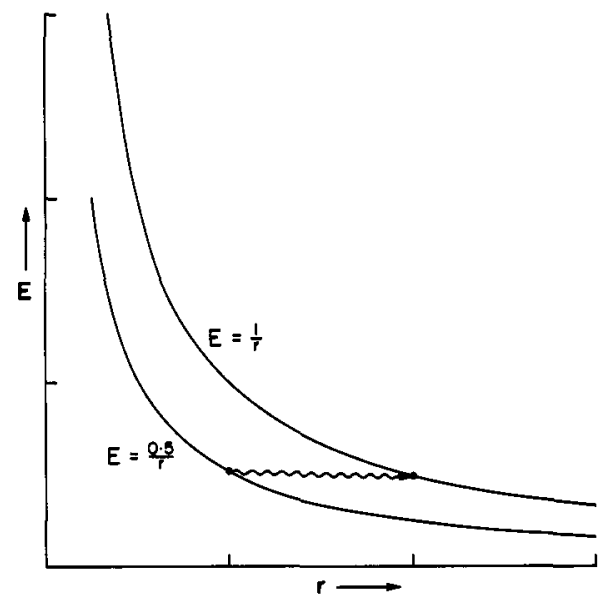

Fig. 2. Schematic potential energy curves showing that transition from $E=0.5 / r$ to $E=1 / r$ involves no change in energy for a doubling of the internuclear separation $r$. 

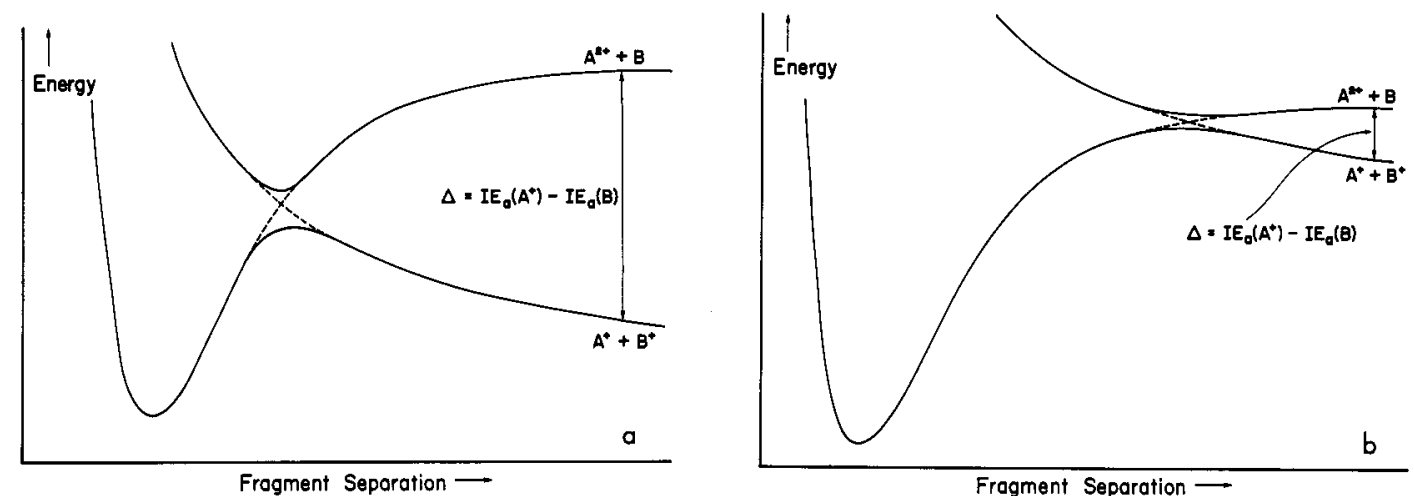

Fig. 3. Schematic potential energy curves for a general $\mathrm{AB}^{2+}$ dication showing avoided crossing between $\mathrm{A}^{2+}+\mathrm{B}$ and $\mathrm{A}^{+}+\mathrm{B}^{+}$diabatic potentials: (a) large $\Delta$; (b) small $\Delta$.

In our preferred view of the dication fragmentation process, we consider the potential energy curve along the reaction coordinate for a dissociating $\mathrm{AB}^{2+}$ dication as arising from an avoided crossing between a repulsive state which correlates with $\mathrm{A}^{+}+\mathrm{B}^{+}$and an attractive state which correlates with $\mathrm{A}^{2+}+\mathrm{B}$ (Fig. 3) (ref. 14). The asymptotic separation $\Delta$ is equal to the difference in ionization energies of $A^{+}$and $B$. When the energy gap $\Delta$ is large, the transition structure occurs early and with a shorter bond length (Fig. 3a).

Conversely, we would predict that small $\Delta$ values will be associated with late transition structures, as displayed in Fig. 3b. It is easy to show that, for sufficiently late transition structures, the bond length in the transition structure is given by

$$
\mathrm{r}_{\mathrm{TS}}=1 / \Delta \text {. }
$$

This formula leads to a transition-structure length of $12.40 \AA$ for $\mathrm{MgH}^{2+}$, in close agreement with the directly calculated $12.45 \AA$. Notice the remarkable feature that this formula requires only knowledge of the ionization energies of the fragments formed.

\section{DICATIONS WITH REMARKABLY SHORT BONDS: SUBSTITUTED METHYLENE DICATIONS ( $\mathrm{HCX}^{2+}$ )}

Dications, despite their extreme coulomb repulsion, often display surprisingly short bond lengths. We have already noted that the $\mathrm{He}_{2}{ }^{2+}$ dication has the shortest known bond.

We have found, in addition, that the $\mathrm{HCX}^{2+}$ dications (substituted methylene dications) represent a family of dications with very short $\mathrm{C}-\mathrm{X}$ bonds (ref. 12). For example. the $\mathrm{C}-\mathrm{F}$ and $\mathrm{C}-\mathrm{Cl}$ lengths calculated for $\mathrm{HCF}^{2+}$ and $\mathrm{HCCl}^{2+}$ (Fig. 4) are, together with those for the corresponding helio trications (see below), the shortest presently known for these types of bonds and substantially less than "normal" $\mathrm{C}-\mathrm{F}$ and $\mathrm{C}-\mathrm{Cl}$ lengths (e.g. in $\mathrm{CH}_{3} \mathrm{~F}$ and $\mathrm{CH}_{3} \mathrm{Cl}$, Fig. 4).

The origin of the bond shortening is clear: the methylene dications have two formally vacant orbitals at the positive carbon which may interact conjugatively or hyperconjugatively with an appropriate $\pi$-electron-donor substituent X (Fig. 5).

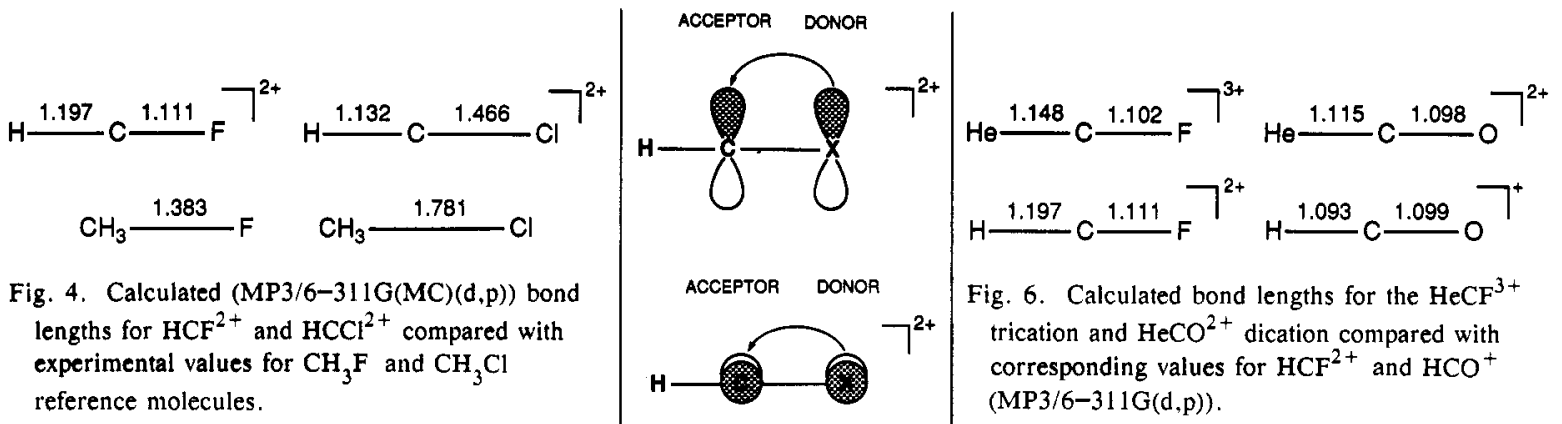

Fig. 5. Conjugative interactions in $\mathrm{HCX}^{2+}$ dications. 
The short $\mathrm{C}-\mathrm{C}$ bond in $\mathrm{CH}_{3}-\mathrm{CH}^{2+}$, originally discovered by Lammertsma et al. (ref. 25), represents an extreme example of hyperconjugation: $1.299 \AA(\mathrm{MP} 3 / 6-31 \mathrm{G}(\mathrm{d}, \mathrm{p}))$ for the length of a formal C-C single bond. Note, however, that there is little or no barrier for rearrangement of $\mathrm{CH}_{3} \mathrm{CH}^{2+}$ to $\mathrm{CH}_{2} \mathrm{CH}_{2}{ }^{2+}\left(\mathrm{ref}^{2}\right.$. $15)$.

As an extension of this work, we have examined related helium-containing ions (ref. 17). We find that the $\mathrm{C}-\mathrm{F}$ bond in the $\mathrm{HeCF}^{3+}$ trication is even shorter than that in the $\mathrm{HCF}^{2+}$ dication (Fig. 6). The $\mathrm{C}-\mathrm{O}$ bond in $\mathrm{HeCO}^{2+}$ is also short, comparable to than in the formyl cation, $\mathrm{HCO}^{+}$.

The $\mathrm{HeCO}^{2+}$ dication is predicted not only to be kinetically stable but also, perhaps surprisingly, thermodynamically stable. Dissociation to $\mathrm{He}^{+\cdot}$ and $\mathrm{CO}^{+\cdot}$ is endothermic by $74 \mathrm{~kJ} \mathrm{~mol}^{-1}$. A possible means of producing $\mathrm{HeCO}^{2+}$, at least in principle, is by $\beta$-decay from the tritioformyl cation (cf ref. 26):

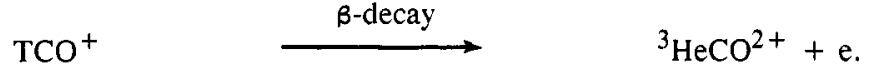

Fragmentation of the $\mathrm{HeCF}^{3+}$ trication is highly exothermic: by $973 \mathrm{~kJ} \mathrm{~mol}^{-1}$. Nevertheless, the barrier to fragmentation (132 $\mathrm{kJ} \mathrm{mol}^{-1}$ ) is sufficiently large that observation should be feasible. The barrier to fragmentation is, in fact, larger than that for the $\mathrm{HCF}^{2+}$ dication $\left(70 \mathrm{~kJ} \mathrm{~mol}^{-1}\right)$, despite less favorable thermodynamics (ref. 17).

\section{STABLE HIGHLY-CHARGED CATIONS: HELIOCARBOCATIONS}

Heliocarbocations have been examined previously by Wilson et al. (ref. 27), Koch and Frenking (ref. 28) and Schleyer (ref. 29). We have been particularly interested in the more highly-charged ions (ref. 16,18).

Our optimized structures for a selection of heliocarbocations are shown in Fig. 7. The most striking feature is that, whereas the mono- and di-cations are only weakly bound, the triheliomethyl trication and tetraheliomethane tetracation have quite short bonds -1.209 and $1.213 \AA$, respectively.

How stable are these species? Our calculations indicate that fragmentation of both the trication and tetracation is highly exothermic - by 1030 and $1605 \mathrm{~kJ} \mathrm{~mol}^{-1}$, respectively. However, the barriers for these processes ( 152 and $72 \mathrm{~kJ} \mathrm{~mol}^{-1}$ ) are sufficiently large that experimental observation should be feasible.

A possible means of preparing the tetraheliomethane tetracation, at least in principle, is via $\beta$-decay from tetratritiomethane:

$$
\mathrm{CT}_{4} \stackrel{\beta \text {-decay }}{\longrightarrow} \quad \mathrm{C}^{3} \mathrm{He}_{4}{ }^{4+}+4 \mathrm{e}
$$

However, the first step in this process is production of the tritiated monoheliomethane monocation. This species is quite unstable and fragments readily (rets 26,30 ):

$$
\mathrm{CT}_{4} \rightarrow\left[\mathrm{CT}_{3}{ }^{3} \mathrm{He}\right]^{+} \rightarrow \mathrm{CT}_{3}^{+}+{ }^{3} \mathrm{He}+\mathrm{e} .
$$

It is rather ironic that it is the instability of the monocation which inhibits the preparation of the (kinetically more stable) tetracation in this manner.

The instability of the monoheliomethane monocation $\left(\mathrm{CH}_{3} \mathrm{He}^{+}\right)$, with a bond length of $2.132 \AA$, may be attributed (ref. 18) to a large energy gap between the helium donor orbital and the methyl cation acceptor orbital. leading to poor donor-acceptor interaction (Fig. 8).

The donor-acceptor interaction in $\mathrm{CH}_{3} \mathrm{He}^{+}$may be improved by lowering the energy of the acceptor orbital. This can be achieved by making the methyl group more pyramidal, i.e. by increasing the $s$ character of the formally vacant orbital. Indeed, by constraining the bond angles to be tetrahedral, the carbon-helium length

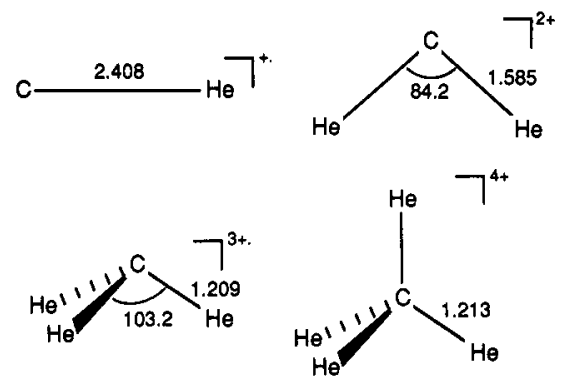

Fig. 7. Optimized structures (MP4/6-311G(d,p)) for $\mathrm{CHe}_{\mathrm{n}}{ }^{\mathrm{n}+}$ cations (from ref. 16).

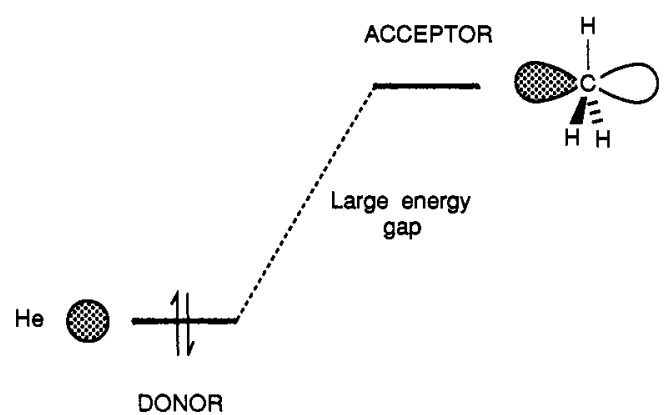

Fig. 8. Orbital interaction diagram for $\mathrm{CH}_{3} \mathrm{He}^{+}$. 


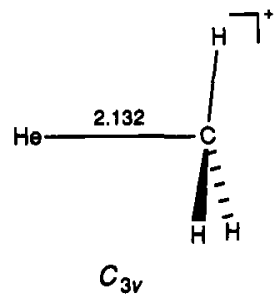

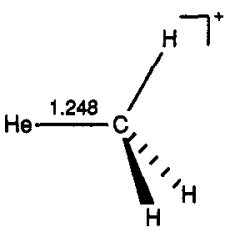

tetrahedral bond angles
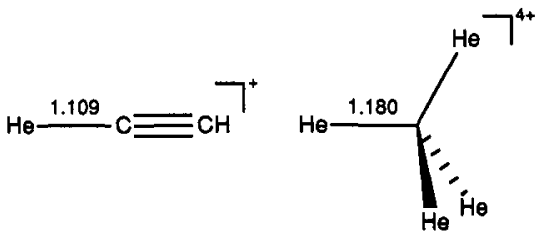

$C_{s}$
$T_{d}$

Fig. 9. Optimized structures (MP3/6-31G(d,p)) for heliocarbocations.

is reduced markedly to $1.248 \AA$ (Fig. 9), even though the energy of this structure is substantially higher than that of the unconstrained form. This result suggests that observation of the monohelio-monocation of a suitably constrained hydrocarbon may be feasible, with preparation again perhaps via $\beta$-decay from the corresponding tritio isotopomer.

For similar reasons, the monohelioacetylene monocation has a relatively short carbon-helium bond $(1.109 \AA)$ in this case the acceptor orbital of the acetylenic moiety being sp-hybridized.

Finally, triheliosubstitution also results in a better matching of donor and acceptor orbitals and again a relatively short carbon-helium bond length $(1.180 \AA$ at MP3/6-31G(d,p)).

\section{CONCLUDING REMARKS}

(1) Multiply-charged cations display interesting structural and energetic features. Despite extreme coulomb repulsion, these species can show very short bonds (e.g. $\mathrm{He}_{2}{ }^{2+}, \mathrm{HCF}^{2+}, \mathrm{HCCl}^{2+}, \mathrm{HeCF}^{3+}$ ) and/or support high charges (e.g. $\mathrm{HeCF}^{3+}, \mathrm{CHe}_{3}{ }^{3+} \cdot, \mathrm{CHe}_{4}{ }^{4+}$ ).

(2) Very long bonds are often found in transition structures for asymmetric fragmentations of multiplycharged ions.

(3) Unrestricted Møller-Plesset perturbation theory is not recommended for the examination of fragmentation of multiply-charged ions in cases where the underlying UHF wavefunction is significantly spin contaminated.

\section{REFERENCES}

1. K. Levsen and H. Schwarz, Mass Spectrom. Rev. 2, 77-148 (1983).

2. E.E. Kingston, J.H. Beynon, T. Ast, R. Flammang and A. Maquestiau, Org. Mass Spectrom. 20, $546-555$ (1985).

3. M. Thompson, M.D. Baker, A. Christie and J.F. Tyson, Auger Electron Spectroscopy. Wiley, New York (1985).

4. P. Millie, I. Nenner, P. Archirel, P. Lablanquie, P. Fournier and J.H.D. Eland, J. Chem. Phys. 84, 1259-1269 (1986).

5. P.v.R. Schleyer, Preprints, Div. Petr. Chem., Am. Chen, Soc. 28 413-418 (1983).

6. W. Koch. F. Maquin, D. Stahl and H. Schwarz. Chimia 39, 376-384 (1985)

7. W. Koch and H. Schwarz, in Structure/Reactivity and Thermochemistry of Ions (P. Ausloos, Ed.), NATO ASI, in press.

8. W. Bouma and L. Radom, J. Am. Chem. Soc. 105, 5484-5486 (1983)

W.J. Bouma and L. Radom, J. Am. Chem. Soc. $107,345-348$ (1985).

B.F. Yates. W.J. Bouma and L. Radom. J. Am. Chem. Soc. 108, 6545-6554 (1986).

M.W. Wong, J. Baker, R.H. Nobes and L. Radom, J. Am. Chem. Soc. 109, 2245-2250 (1987).

M.W. Wong, B.F. Yates, R.H. Nobes and L. Radom, J. Am. Chem Soc. 109. 3181 1-3187 (1987).

P.M.W. Gill and I. Radom. Chem. Phys. Lett. 132, 16-22(1986).

4. P.M.W. Gill and L. Radom. Chem. Phys. Lett, 136, 294-298 (1987).

5. R.H. Nobes, M.W. Wong and L. Radom. Chem. Phys. Lett. 136, $299-302$ (1987).

. M.W. Wong, R.H. Nobes and L. Radom, J. Chem. Soc., Chem. Commun. 233-234 (1987).

M.W. Wong, R.H. Nobes and L. Radom, Rapid Commun. Mass Spectrom. 1, 3-5 (1987).

M.W. Wong, H.B. Bürgi and L. Radom, to be published.

W.J. Hehre, L. Radom. P.v.R. Schleyer and J.A. Pople, Ab Initio Molecular Orbital Theory. Wiley, New York (1986).

H. Yagisawa, H. Sato and T. Watanabe, Phys. Rev. A16. 1352-1357 (1977).

M. Guilhaus, A.G. Brenton, J.H. Beynon, M. Rabrenovic and P.v.R. Schleyer, J. Phys, B 17, L605-L610 (1984).

N.C. Handy, P.J. Knowles and K. Somasundram. Theor. Chim. Acta 68, 87-100 (1985).

H.B. Schlegel, J. Chem. Phys. 84, 4530-4534 (1986).

R.H. Nobes, J.A. Pople, L. Radom, P.J. Knowles and N.C. Handy, Chem. Phys. Lett. in press.

25. K. Lammertsma, M. Barzaghi, G.A. Olah, J.A. Pople, A.J. Kos and P.v.R. Schleyer, J. Am. Chem. Soc. 105, 52525257 (1983).

26. F. Cacace, Adv. Phys. Org. Chenn. 8, 79-149 (1970).

27. S. Wilson and S. Green. J. Chem. Phys. 73. 419-424 (1980); D.L. Cooper and S. Wilson, Mol, Phys. 41, 161-172 (1981).

28. W. Koch and G. Frenking. J. Chem. Soc. Chem. Commun. 1095-1096 (1986); W. Koch and G. Frenking, Int. J. Mass Spectrom. Ion Proc. 74. 133-136 (1986).

29. P.v.R. Schleyer, Adv. Mass Spectrom. 287.301 (1985).

30. A.H. Snell and F. Pleasonton, J. Phys. Chem. 62, 1377-1382 (1958). 